\title{
Optimization of the illumination optics for gap flow velocity measurements in turbo machines
}

\author{
Raimund Schlüßler, Christian Blechschmidt, Andreas Fischer, Jürgen Czarske \\ Technische Universität Dresden, Laboratory for Measuring Techniques, Dresden Germany, \\ E-Mail: raimund.schluessler@tu-dresden.de
}

\begin{abstract}
:
Understanding and designing the tip leakage flow in turbo machines is one important point for increasing their efficiency. Therefore optical flow velocity measurements in the less than $1 \mathrm{~mm}$ wide tip gap are necessary. These measurements are very challenging because disturbing light reflections e.g. from the rotor blade tip are increasing the measurement uncertainty. Hence, the disturbing light reflections need to be reduced.

Using diffraction theory it was possible to optimize the parameters of the illumination beam. Combined with an absorptive coating of the rotor tip, this results in a reduction of the disturbing light power by a factor of more than 100 compared to the previously used setup. This enables optical 3-dimensional-3component flow velocity measurements in sub millimeter gaps.
\end{abstract}

Key words: Optical flow velocity measurement, sub millimeter gaps, optimized laser beam, diffraction theory

\section{Introduction and State-of-the-art}

In order to increase the efficiency of turbo machines, it is important to know the flow characteristics inside the machines. Especially the tip leakage flow in the down to $900 \mu \mathrm{m}$ small gap between the rotor blade tip and the turbine housing is of high interest. The tip leakage flow induces turbulence, which decreases the efficiency of the turbo machine [1]. It was shown, that optical flow measurement techniques are a suitable tool for measuring the flow velocity inside turbo machines [2].

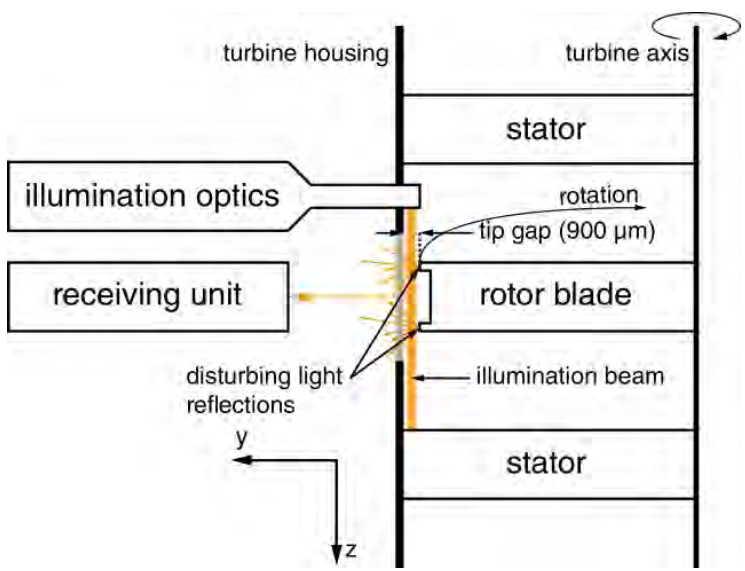

Fig. 1: Scheme of the measurement setup.
However, optical flow velocity measurements in small gaps are a very challenging task, because disturbing light reflections from many different sources can occur. As shown in the scheme of the measurement setup in Fig. 1, the main sources for such disturbances are the rotor blade tip and the turbine housing, as they potentially reflect light from the illumination beam directly into the observation aperture. For some turbine configurations also additionally reflections from the stator can occur.

Recent optical flow velocity measurements in a turbo machine using FM-DGV were able to measure the flow velocity in the gap between the rotor blade tip and the turbine housing with three components [2]. However, the measurement uncertainty suffered from a high interfering light power resulting from disturbing light reflections at the turbine blades. The acquired tangential flow velocity component is depicted in Fig. 02. At the white spots in the figure, the chosen threshold of the interfering light power was exceeded and the velocity values had to be discarded. Here, two principle effects of a high interfering light power can be differentiated: 


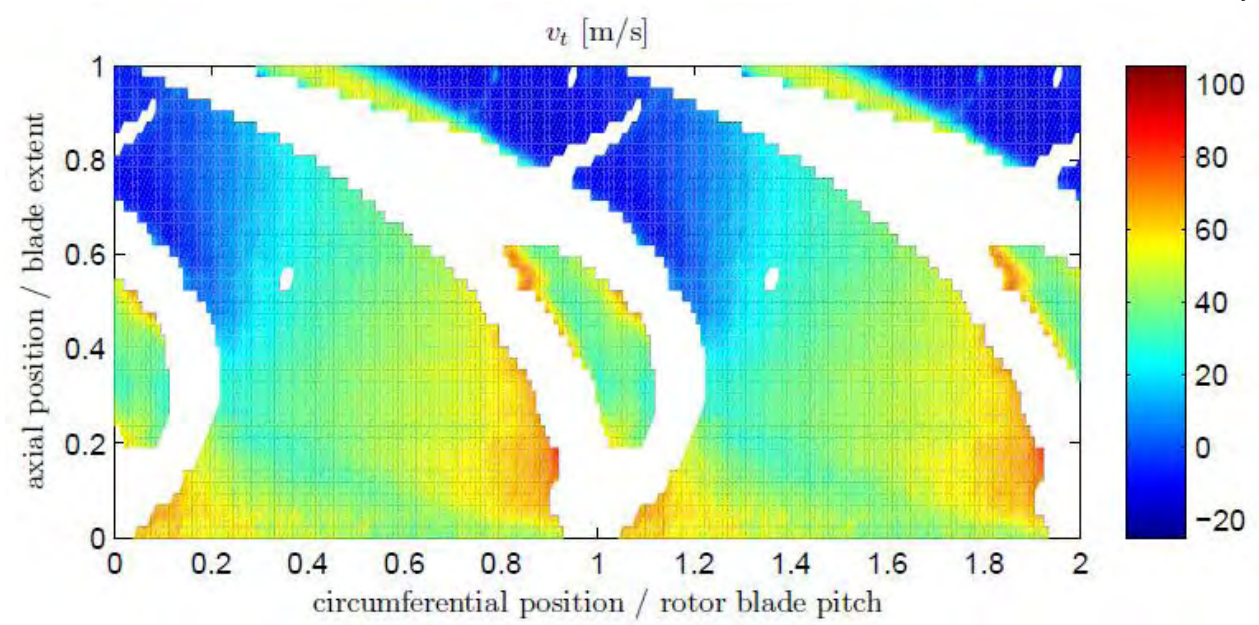

Fig. 2: Measured tangential velocity in the gap between the rotor blade tip and the turbine housing [2]. At the white spots the photo detectors were saturated due to a high interfering light power or the interfering power exceeded the chosen threshold.

Saturation of the photo detectors: If the interfering light power is higher than approximately $20 \mathrm{nW}$ the photo detectors are saturated. This error cannot be corrected by signal analysis and thus it is not possible to acquire valid flow velocity values. A saturation of the photo detectors mainly occurs at positions where the width of the gap is very low and the illumination light is directly reflected into the observation aperture, e.g. by the rotor blade tip.

Increase of the measurement uncertainty: If the interfering light power does not saturate the photo detectors, it is still possible to acquire valid velocity values. However, the systematic as well as the random error will be increased, as the disturbing light interferes with the used stray light from the tracer particles [3].

Thus, the most important task is to the prevent the photo detectors from saturating in order to enable flow velocity measurements at the rotor tips. Furthermore, the interfering light power should be lower than the expected scattered light power $P_{p}$ of $0.5 \mathrm{nW}$, in order to minimize the measurement uncertainty.

In this paper, we present two different approaches to reduce the interfering light power. In the first section we show how to optimize the illumination beam profile, in order to minimize the light power on the gap edges. Subsequently the effect of an absorptive coating at the blade tips is analyzed. In conclusion we show flow measurements in a simplified turbine model to proof the reduction of the stray light power.

\section{Solution}

To prevent a saturation of the photo detectors, the interfering light power must be reduced. This can be achieved by optimizing the

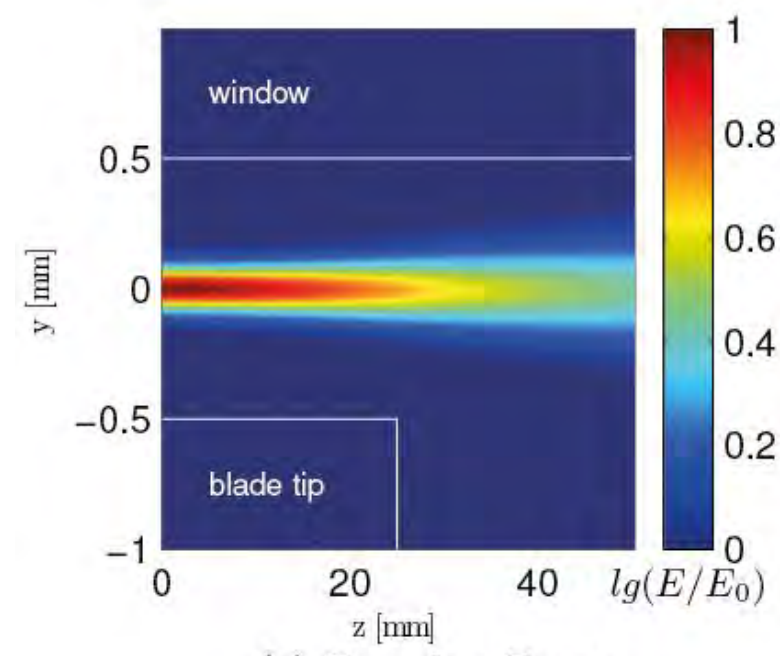

(a) Gaussian Beam

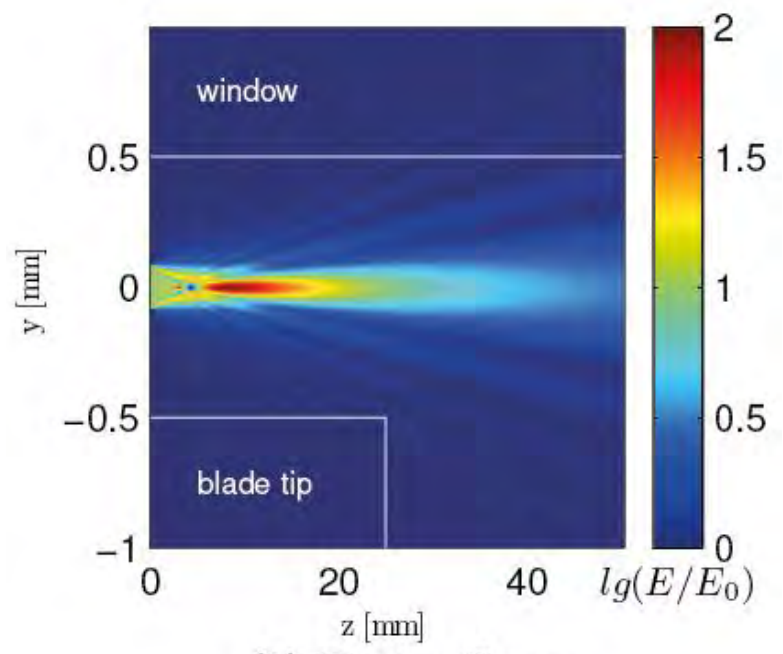

(b) Flattop Beam

Fig. 3: Example of the beam propagation of (a) a Gaussian beam and (b) a beam with Flat Top profile. The light power on the rotor blade and turbine housing is $2.5 \times 10^{13}$ times higher for the Flat Top profile than for the Gaussian beam shape (cp. Fig. 4). 
illumination beam shape to reduce the amount of light hitting the edges of the gap. Furthermore the reflectivity of the illuminated surfaces can be reduced, so that a lower amount of light is reflected.

\section{Beam shaping}

For the optimal illumination beam shape the interfering light power $P_{w}$ on the gap walls has to be minimal. The approach of using a SuperGaussian beam shape is very promising, because the light power is redistributed from the border to the center of the beam and a very sharp edge of the beam profile occurs. This should reduce the interfering light power $P_{w}$ on the gap walls. However, this is only true for the focus point. As the beam propagates, it will diverge and potentially illuminate the gap walls. Hence, the interfering light power $P_{w}$ for different Super-Gaussian illumination beam shapes

$$
I(\rho)=I_{0} e^{-2\left(\rho / w_{0}\right)^{p}}
$$

of the order $p$ with the beam waist radius $w_{0}$ has to be calculated. Therefore, the intensity distribution has to be known. In order to calculate the intensity distribution at $z>0$ for a given intensity distribution at the focus point $(z=0)$, the Split-step beam propagation method is used [4]. It is assumed, that the wave fronts of the illumination beam are plane in the focus point, which results in the lowest divergence. Whether the regarded intensity distribution is achievable in the experiment is not considered. Thus, the calculation provides the best result theoretically possible for each beam shape.

In Fig. 3 two examples for the propagation of a beam with a Gaussian $(p=2)$ and a Flattop profile $(p=100)$ are shown. The light power of the Flattop profile at the focus point is concentrated at the center of the gap. This results in a lower illumination of the gap walls for the focus point. However, the divergence of the Flattop profile beam is much higher than for the standard Gaussian beam. Depending on the length and width of the gap, this results in a higher interfering light power at the gap walls.

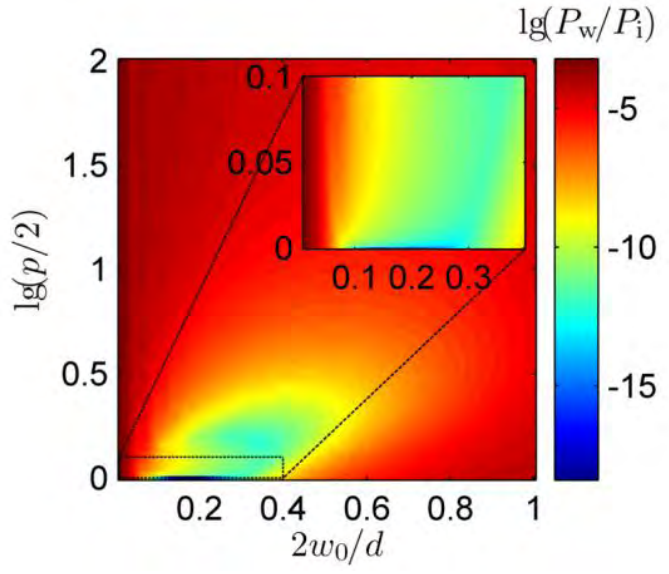

Fig. 4: Simulation of the light power $P_{w}$ on the gap walls for different orders $p$ of super Gaussian profiles and beam waist radii $w_{0}$ normalized to the gap width $d$. An optimum was found for $p=2$ and $w_{0}=w_{0, \min }$.

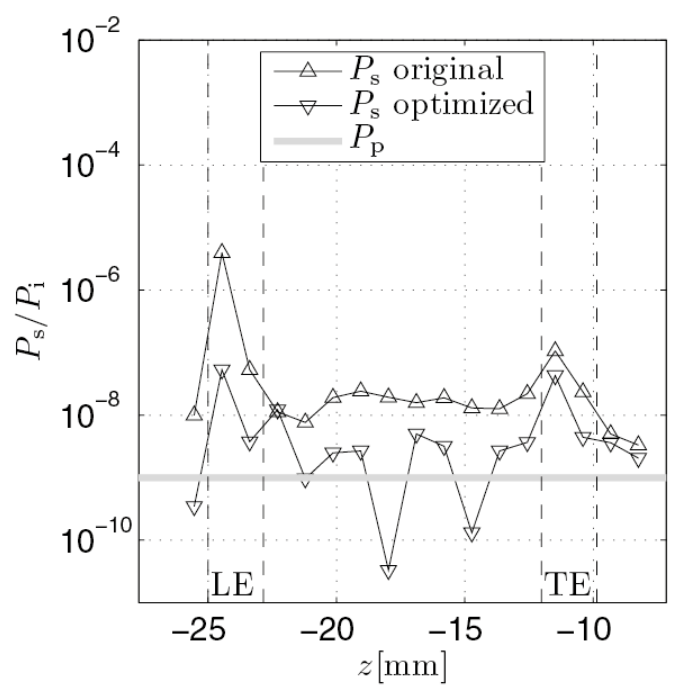

Fig. 5: Measurement of the interfering light power $P_{s} \sim P_{w}$ in relation to the incident light power $P_{i}$ for the original setup with $w_{0} \approx 2 w_{0, \min }$ and the optimized setup with $w_{0}=w_{0, \min }$ along the beam propagation direction $z$. The highest interfering light power occurs for the leading edge of the rotor blade tip at $z=-24 \mathrm{~mm}$. 


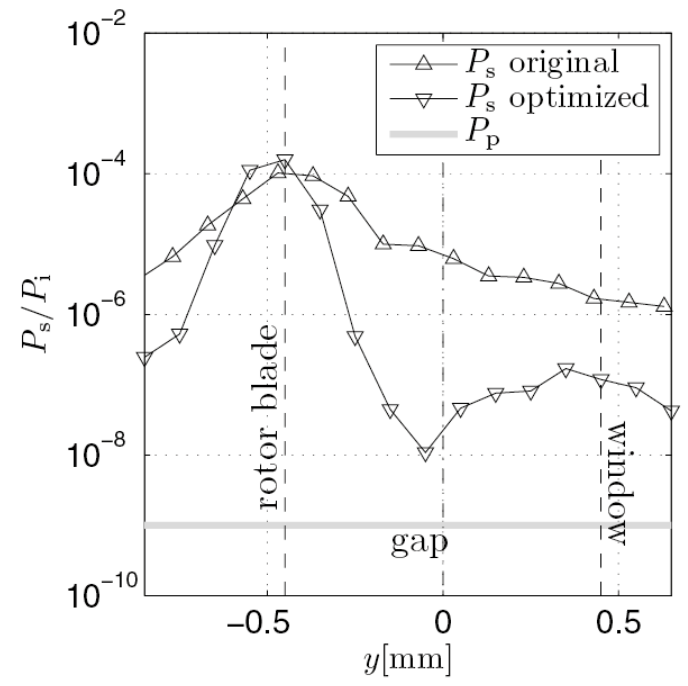

Fig. 6: Measurement of the interfering light power $P_{s} \sim P_{w}$ in relation to the incident light power $P_{i}$ for the original setup with $w_{0} \approx 2 w_{0, \min }$ and the optimized setup with $w_{0}=w_{0, \min }$ at the leading edge of the rotor blade tip. The illumination beam was traversed along the $y$ direction normal to the turbine axis. The interfering light power was reduced by nearly factor 100 at the center of the tip gap.

The calculated interfering light power $P_{w}$ for different Super-Gaussian beam shapes is shown in Fig. 4. For this calculation the parameters of the real tip gap with a length $l$ of $5 \mathrm{~cm}$ and a width $d$ of $900 \mu \mathrm{m}$ are used. The interfering light power is normalized to the incident light power $P_{i}$. The minimum of $P_{w}$ is found for $p=2$. Contrary to the first assumption, the classic Gaussian beam of the order 2 provides the lowest interfering light power for the given parameters of the gap. This implies, that is not reasonable to use a SuperGaussian beam profile for the illumination beam. Instead, the beam waist radius $\mathrm{w}_{0}$ of the standard Gaussian beam has to be optimized. It is possible to find an analytical expression for the optimal beam waist radius

$$
w_{0, \min }=M \sqrt{\frac{\lambda l}{2 \pi}}
$$

with the wavelength $\lambda$ of the used light source, the beam quality product $M$ and the length $l$ of the gap. The beam waist radius for the regarded gap is $87 \mu \mathrm{m}$. This result is in agreement with the numerical analysis in Fig. 4.

For evaluating the decrease of the interfering light power due to the optimized illumination beam, measurements of the interfering light power $P_{s}$ were performed on a model of the rotor blade tip gap. Because of the scattering and reflection of the illumination beam on the gap walls, only the fraction $P_{s} \sim P_{w}$ of the total light power $P_{w}$ on the gap walls enters the observation aperture. The interfering light power $P_{S}$ is measured with a camera and is shown in Fig. 5 and Fig. 6. In Fig. 5 the interfering light power is plotted versus the illumination beam direction $z$. The highest power is measured at the leading edge of the rotor blade tip at $z=-24 \mathrm{~mm}$. Hence, in Fig. 5 the interfering light power was measured at $z=-24$ and the illumination beam was traversed along the $y$-direction normal to the turbine axis. For $y=-0.5 \mathrm{~mm}$ the illumination beam hits the rotor blade tip and the resulting light power is equal for the original and the optimized beam. This is due to the fact, that at the blade tip a direct reflex of the illumination beam is reflected into the observation aperture and the total light power is not changed by the optimization of the illumination beam profile. At the center of the gap the interfering light power is reduced by a factor of nearly 100 compared to the original used illumination beam. This is a significant improvement and shows, that the optimization is useful. However, the ratio of the interfering light power to the incident light power is still higher than the ratio of $10^{-9}$ of the expected mean scattered light power to the incident light power. This makes a further reduction of the interfering light power necessary.

\section{Absorptive coating}

A further reduction of the interfering light power $P_{s}$ can be achieved by lowering the reflection coefficient of the gap walls [2]. This is realized by an absorptive coating of the rotor blade tip and the turbine housing or by anodizing the surfaces in case the materials used are suitable, e.g. aluminum.

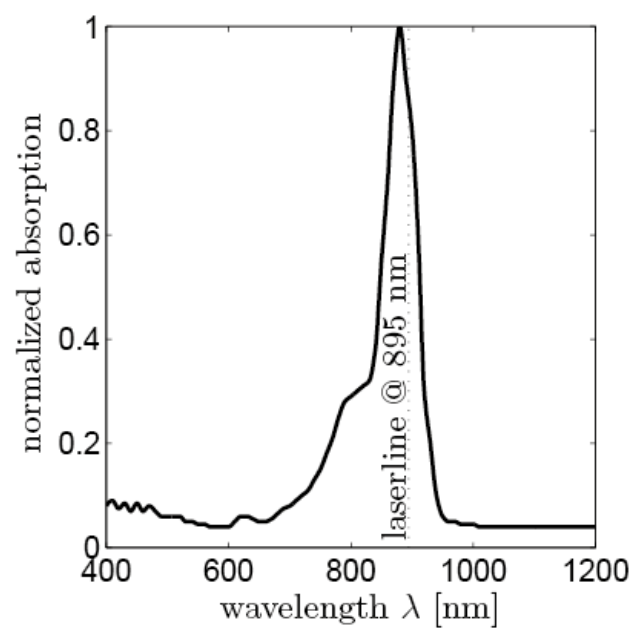

Fig.6: Absorption spectrum of the used dye Spectre340. The absorption maximum is near the used laser wavelength of $895 \mathrm{~nm}$. 


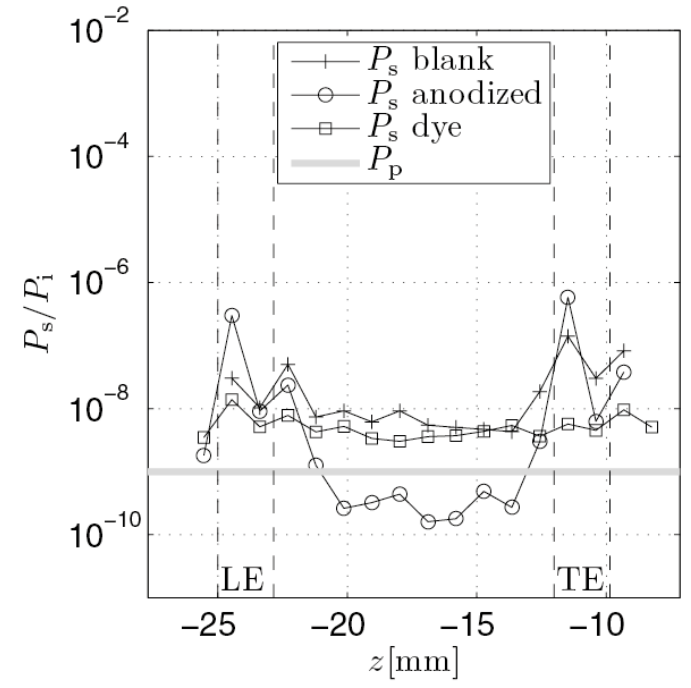

Fig. 7: Measurement of the interfering light power along the beam propagation direction $z$ for different surface treatments. At the center of the gap

The dye used for the coating must absorb light with the wavelength $\lambda$ of the illumination beam. In our case the wavelength is $895 \mathrm{~nm}$. As this is near infrared, the selection of suitable dyes is limited. In Fig. 6 the absorption spectrum of the used dye Spectre 340 from the Co. EPOLIN is shown [5]. The absorption maximum is near $895 \mathrm{~nm}$. Hence, the dye is suitable for our application. We tested the reflected light power for three different rotor blade tip surfaces: blank aluminum, anodized aluminum and blank aluminum coated with the absorptive dye. The interfering light power $P_{s}$ was measured with the same setup used for measuring the reduction due to the optimized beam shape explained in the previous section.

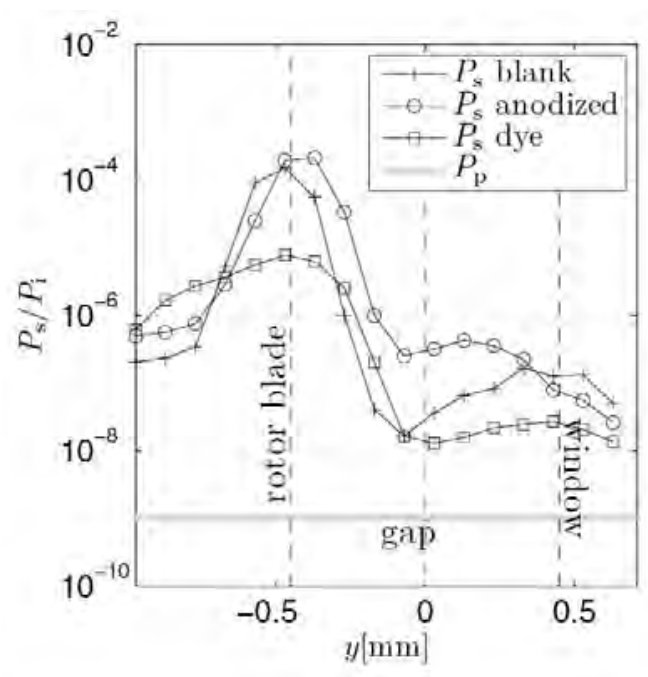

Fig. 8: Comparison of the interfering light power $P_{s}$ in relation to the incident light power $P_{i}$ for an uncoated rotor blade tip and a blade tip coated with Spectre340. The interfering light power was reduced by a factor of 100 at the center of the gap.
The measured interfering light power $P_{s}$ for rotor blade tips with different surface treatments is depicted in Fig. 7 and Fig. 8. Fig. 7 shows the interfering light power along the beam propagation direction. For $z=-17 \mathrm{~mm}$ in the center of the gap the absorptive dye shows a reduction of the interfering light power by factor 2 compared to the blank aluminum, but the anodized surface shows a further reduction by factor 100 . However, at the leading edge (LE) and tailing edge (TE) of the rotor blade shown in Fig. 8 this behavior changes. Here, the interfering light power due to the absorptive dye is factor 100 smaller than due to the anodized surface. This may result from a varying thickness of the dye because of its high surface tension.

As a result, a combination of an anodized surface with an absorptive coating leads to the lowest interfering light power at all positions in the gap. This combination should be able to reduce the light power by an factor of 100 compared to an untreated blank aluminum surface. Hence, flow velocity measurements in the narrow tip gap are possible. We will proof this assumption in the next section by velocity measurements at a model of the turbine tip gap.

\section{Flow Velocity Measurements}

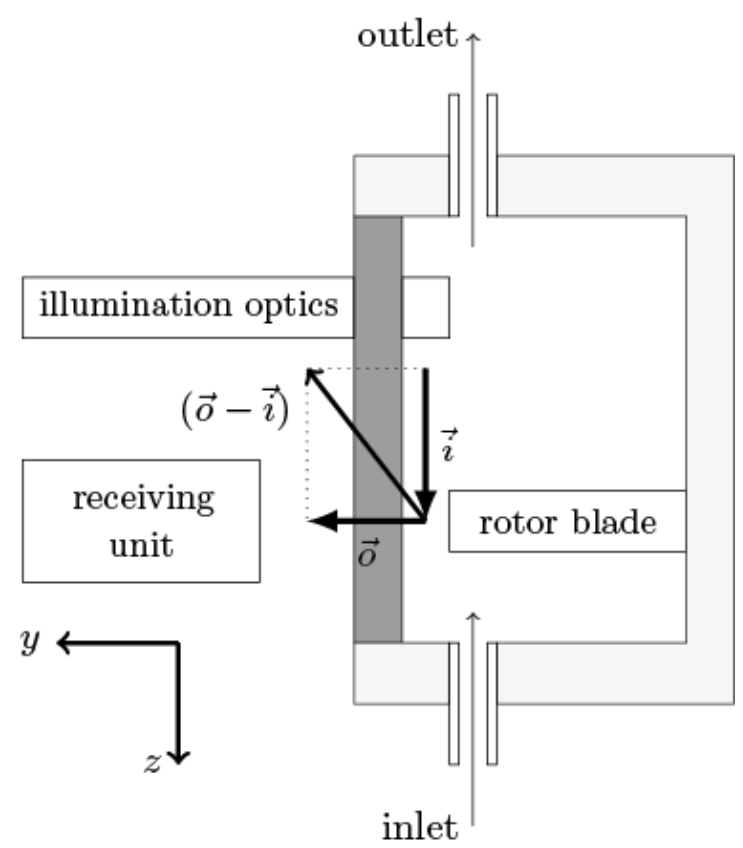

Fig. 9: Scheme of the setup for the flow velocity measurement.

For the flow velocity measurements in the small gap we used a very simple model of a turbo machine. The scheme of the setup if shown in Fig. 9. We added an inlet and an outlet to the setup in Fig. 1 and applied an air flow in negative $z$-direction driven by a compressor. The measured velocity component is $(\vec{o}-\vec{\imath})$ as shown in the scheme. 


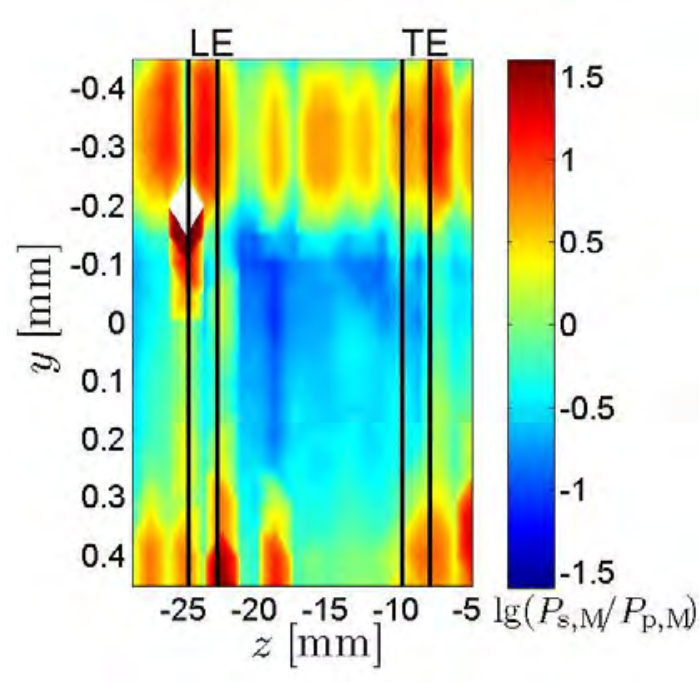

Fig. 10: Mean interfering light power $P_{\mathrm{S}, \mathrm{M}}$ normalized to the mean scattered light power $P_{\mathrm{p}, \mathrm{M}}$.

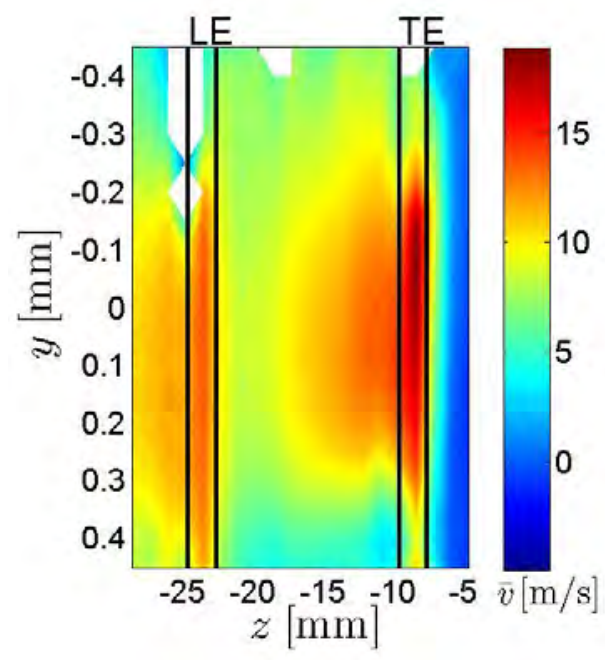

Fig. 11: Measured mean flow velocity component.

In Fig. 10 the interfering light power for different positions $y$ of the beam in the gap is shown. In the range from $y=0$ to $y=0.25 \mathrm{~mm}$ the interfering light power is less than 3 times higher than the used scattered light power and no saturation of the photo detectors occurs. Hence, a measurement of the flow velocity is possible in this area. The results of the flow velocity measurement are shown in Fig. 11. The qualitative characteristics of the acquired results meets the expectations. The flow direction is correct and the magnitude of the velocity rises at the leading and trailing edge of the rotor blade in the center of the gap. Also for positions closer to the surface of the rotor blade or the turbine housing, the magnitude of the flow velocity decreases. However, reference measurements are necessary for a quantitative analysis, which have to be conducted yet.

\section{Summary and outlook}

By the optimization of the illumination beam shape the interfering light power was reduced by a factor 100 compared to the previously used setup. Furthermore, the surface treatment of the rotor blade tip leads to an additional reduction by also a factor 100 at the narrowest locations of the gap. This results in a total decrease of the interfering light power by more than factor 100 at the most critical spots, e.g. the leading edge of the rotor blade tip. A further reduction of the interfering light power may be achieved by evaluating the polarization of the received light, to separate the used scattered light from the reflected interfering light.

Still, these results need to be verified by flow velocity measurements in a real turbo machine. Also, the sensitivity of the optimized illumination optics to vibrations of the turbine and to temperature variations has to be analyzed.

However, the results presented in this paper enable 3-dimensional-3-component optical flow velocity measurements in sub millimeter gaps.

\section{References}

[1] C. Camci, D. Dey, L. Kavurmaciogly: Rotor-Tip Leakage Part I - Basic Methodology, Journal of Engineering for Power 104(1): 154-161, 1982.

[2] A. Fischer, J. König, L. Büttner, J. Czarske, C. Rakenius and H.-P. Schiffer: Tip clearance flow field measurements at at turbine rotor with squealers, 16th Int. Symp. on Applications of Laser Techniques to Fluid Mechanics, 2012.

[3] A. Fischer, D. Haufe, L. Büttner, J. Czarske: Scattering effects at near-wall flow measurements using Doppler global velocimetry, Applied Optics, 50, 4068-4082, 2011.

[4] T.-C. Poon, T. Kim: Engineering optics with MATLAB, World Scientific, 2006.

[5] EPOLIN: Spectre340 infrared absorbing ink, Epolin Inc., Technical Data Sheet, 2011. 